07

\title{
Формирование изображения краевой дислокации при аномальном прохождении рентгеновского излучения
}

\author{
() И.А. Смирнова ${ }^{1}$, Е.В. Шулаков ${ }^{2}$, Э.В. Суворов ${ }^{1}$ \\ ${ }^{1}$ Институт фозики твердого тела РАН, \\ Черноголовка, Россия \\ ${ }^{2}$ Институт проблем технологии микроэлектроники РАН, \\ Черноголовка, Россия \\ E-mail: irina@issp.ac.ru
}

Поступила в Редакцию 18 апреля 2019 г.

В окончательной редакции 18 апреля 2019 г.

Принята к публикации 18 апреля 2019 г.

Рассмотрены особенности формирования изображения секционных и проекционных топограмм краевой дислокации перпендикулярной поверхности кристалла в случае аномального прохождения рентгеновского излучения. Экспериментальные изображения анализируются с использованием численного моделирования дифракционного эксперимента. Предложен новый механизм формирования изображения дефектов расположенных вблизи выходной поверхности кристалла. Отмечены отличия топографических изображений дислокации от розеток локальных разориентаций отражающих плоскостей.

Ключевые слова: рентгеновская топография, дефекты кристаллической структуры, дифракционный контраст дефектов, уравнения Такаги.

DOI: 10.21883 /FTT.2019.08.47977.453

\section{1. Введение}

Наличие дефектов в кристаллах определяет их механические и электрические свойства. Методы рентгеновской топографии, обладают высокой чувствительностью к нарушениям идеальности кристаллической решетки и позволяют получить информацию об объемном распределении дефектов без нарушения целостности и качества образца. Для исследования реальной структуры полупроводниковых, ионных или металлических кристаллов используются различные методы рентгеновской топографии. В первую очередь это метод сканирования в условиях аномального прохождения рентгеновского излучения, метод широко расходящегося пучка, метод Шульца, Фудживара, Берга-Баррета, метод секционной топографии. Однако для большинства экспериментальных ситуаций возможен лишь качественный анализ наблюдаемого изображения дефектов. Количественный анализ рентгеновского дифракционного изображения дефектов в кристаллах требует математического моделирования эксперимента.

Единичные локальные дефекты структуры можно обнаруживать и успешно идентифицировать методом секционной топографии $[1,2]$. Метод состоит в том, что перед кристаллом устанавливается щель, ограничивающая расходимость первичного пучка. В этом случае падающий пучок засвечивает в кристалле треугольник рассеяния, ограниченный векторами падающей $\mathbf{K}_{0}$ и отраженной $\mathbf{K}_{h}$ волн (палатка Бормана).

В секционной топографии достигнуты значительные успехи в интерпретации экспериментальных изображений дефектов в кристаллах. В работе [3] рассмотрены механизмы формирования изображения дислокаций в секционной топографии. Было установлено, что при рассеянии рентгеновских лучей в ближнем поле дислокаций возникают новые рентгено-оптические эффекты аналогичные внутреннему отражению от поверхностей раздела $[4,5]$ и дифракционному волноводному эффекту $[6,7]$, впервые наблюдавшихся для дефектов упаковки. Как отмечалось ранее, эти эффекты, аналогичны интерференционному сжатию рассеянной волны в кристаллах с ультразвуковым полем [8]. Было установлено, что они играют определяющую роль в формировании дифракционного изображения, связанного с быстро изменяющимся упругим полем вблизи ядра дислокации. Сопоставление изображений с разной ориентацией дислокации в треугольнике рассеяния позволяет оценить роль различных эффектов, определяемых интерференцией волновых полей, образованных идеальной матрицей и локальной сильно искаженной областью кристалла [9-12].

Рассмотрим симметричную дифракцию на прохождение в геометрии по Лауэ для сферической волны. При динамической дифракции рентгеновского излучения в совершенном кристалле распространяются две волны, отвечающие разным листам дисперсионной поверхности. Поглощение излучения для этих волн различно и описывается интерференционными коэффициентами поглощения $P_{1,2}$

$$
P_{1,2}=P\left[1 \pm \varepsilon\left(1-s^{2}\right)^{1 / 2}\right] .
$$

Здесь $P=\mu t / \cos \theta, \mu-$ линейный коэффициент фотоэлектрического поглощения. Параметр нормального поглощения $P$ описывает только общее ослабление интенсивности на топограмме и не влияет на особенно- 
сти распространения излучения в кристалле. Параметр $\varepsilon=\chi_{\text {hi }} / \chi_{\text {oi }}$, где $\chi_{\text {hi }}$ и $\chi_{\text {oi }}-$ мнимые коэффициенты поляризуемости кристалла для отраженной и преломленной волны. Параметр $s=\operatorname{tg} \varphi / \operatorname{tg} \theta$ описывает положение точки наблюдения на выходной поверхности внутри треугольника рассеяния и изменяется от -1 до $1, \varphi-$ направление в точку наблюдения, $\theta-$ угол Брэгга. Условие $s=0$ отвечает центру треугольника рассеяния. При смещении точки наблюдения к периферийной области $(|s|$ изменяется от 0 до 1) интерференционное поглощение уменьшается и на краях треугольника рассеяния равно нормальному поглощению $P$.

Параметр $\varepsilon$ зависит от материала кристалла и отражения. В алмазоподобных структурах и медном излучении все отражения с четными индексами отражений $(h, k, l)$ имеют большие значения $\varepsilon$ чем с нечетными. При увеличении индексов отражения параметр $\varepsilon$ уменьшается, и зависимость $\varepsilon$ от $\left(h^{2}+l^{2}+k^{2}\right)^{1 / 2}$ образует для четных и нечетных индексов две непересекающиеся ветви. Для сильных отражений $\operatorname{Si}(220)$ и $\operatorname{Si}(111) \varepsilon=0.97$ и $\varepsilon=0.61$, соответственно. Подставляя эти значения в формулу (1) для отражения (220) и $s=0$ видно, что $P_{1}=1.97 P$ отвечает сильно поглощающейся волне и соответственно $P_{2}=0.03 P$ слабо поглощающейся. Первая волна затухает, а вторая имеет аномально малое поглощение. При увеличении толщины кристалла остается только слабо поглощающаяся волна и реализуется случай аномального прохождения рентгеновского излучения (эффект Бормана). Для отражения (111) этот эффект выражен более слабо.

В работе исследовано формирование изображения краевой дислокации перпендикулярной входной поверхности кристалла в случае аномального прохождения рентгеновского излучения в методах секционной топографии и топографии сканирования (проекционная топография).

\section{2. Методика эксперимента и экспериментальные данные}

Все эксперименты были выполнены на лабораторном микрофокусном источнике неполяризованного излучения с оптическим размером фокуса $28 \times 30 \mu \mathrm{m}$. Топограммы снимались на спектрометре A-3 фирмы Rigaku Denki. Схема секционной и проекционной топографии показана на рис. 1. На кристалл падает расходящийся пучок рентгеновского излучения, угловой спектр которого ограничивается щелью $S_{2}=10 \mu \mathrm{m}$ и оценивается как 14 угловых секунд. В лабораторных условиях щель $S_{2}$ можно считать протяженным некогерентным источником [13]. Третья щель имела размер около $2 \mathrm{~mm}$. Она располагалась непосредственно перед фотопластинкой и служила для уменьшения фона рассеянного излучения на элементах оптической схемы, а также для отсечения проходящего пучка. Секционные топограммы получают при неподвижном кристалле и фотопластинке.

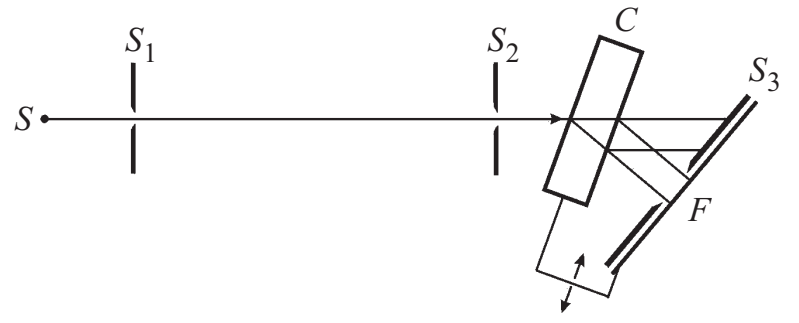

Рис. 1. Схема эксперимента. $S-$ источник рентгеновского излучения; $S_{1}, S_{2}, S_{3}$ - первая, вторая и третья щели; $C$ - кристалл; $F$ - фотопластинка. Расстояние $S S_{1}=55 \mathrm{~mm}$, $S_{1} S_{2}=260 \mathrm{~mm}, S_{2} C=35 \mathrm{~mm}, C P=15 \mathrm{~mm}$.

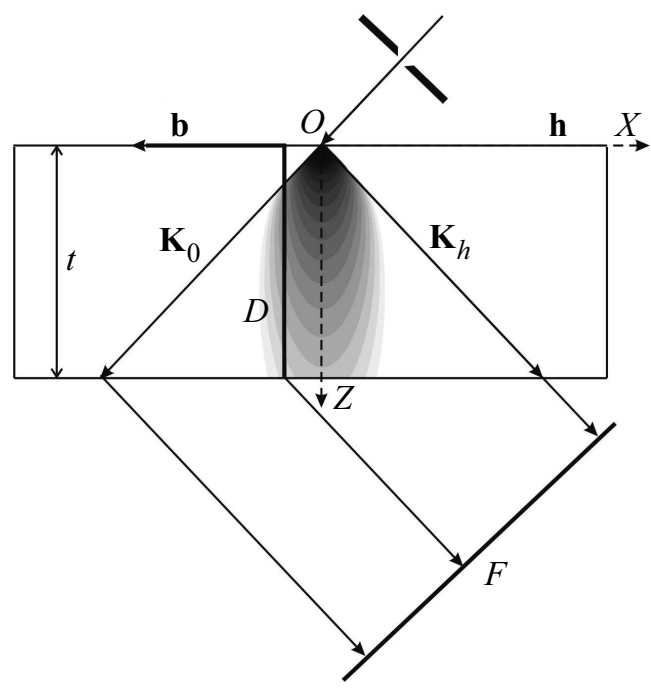

Рис. 2. Геометрия дифракции. $D-$ линия дислокации, $\mathbf{b}-$ вектор Бюргерса, $\mathbf{K}_{0}$ и $\mathbf{K}_{h}-$ волновые вектора падающей и рассеянной волн. $O$ - точка входа излучения в образец, $F$ - плоскость регистрации.

Проекционные топограммы получают при одновременном движении кристалла и фотопластинки параллельно поверхности образца.

Геометрия дифракции в случае аномального прохождения рентгеновских лучей дана на рис. 2. Излучение падает на кристалл под углом Брэгга $\theta$. Ось $X$ в лабораторной системе координат направлена вдоль вектора обратной решетки и совпадает с направлением сканирования. Ось $Y$ перпендикулярна плоскости рисунка. Ось $Z$ направлена вглубь кристалла вдоль направления [110],

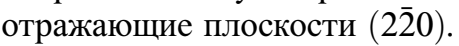

Все топограммы, приведенные в статье, получены на одном образце толщиной $t=1810 \mu \mathrm{m}$ от одной и той же дислокации и излучении $\mathrm{Cu} K_{\alpha 1}$. Ширина треугольника рассеяния на выходной поверхности образца равна $L_{b}=2 t \operatorname{tg} \theta_{B}=1585 \mu \mathrm{m}$. Для данной толщины кристалла интерференционные коэффициенты поглощения $P_{1}=55.9, P_{2}=0.88(P=28.4)$, и в кристалле реализуется аномальное прохождение рентгеновского излучения для второго поля. В этом случае интенсивность на выходной поверхности кристалла имеет заметную величину в более узкой области и равна $331 \mu \mathrm{m}$. Назовем 


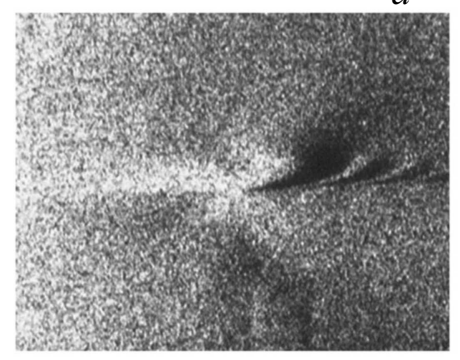

$d$

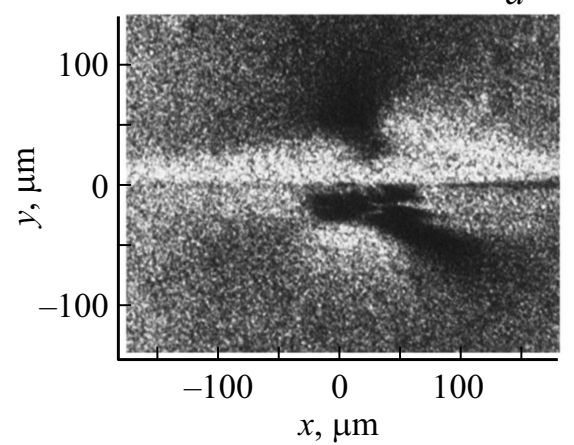

$b$

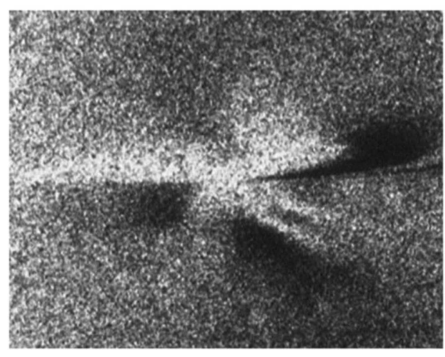

$e$

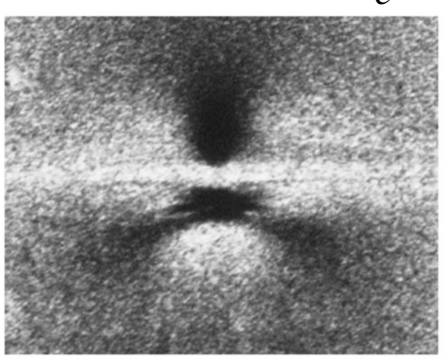

$c$

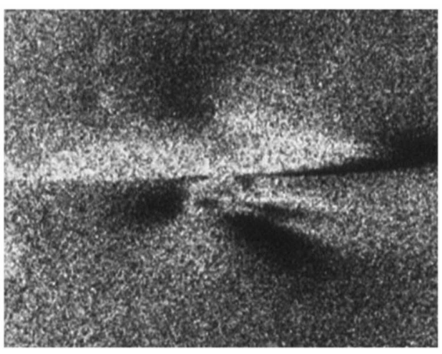

$f$

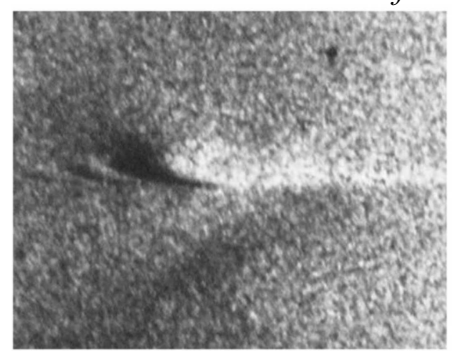

Рис. 3. Фрагменты секционных изображений краевой дислокации, $\operatorname{Si}(2 \overline{2} 0)$. Смещения образца относительно точки $O: a)-100 \mu \mathrm{m}$, b) $-60 \mu \mathrm{m}, c)-40 \mu \mathrm{m}, d)-20 \mu \mathrm{m}, e)-0 \mu \mathrm{m}, f)-100 \mu \mathrm{m}$.

$a$

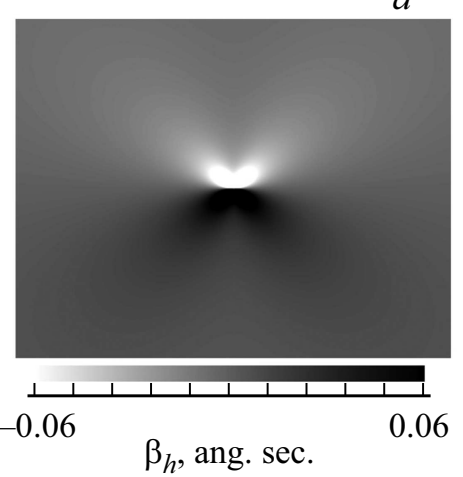

$b$

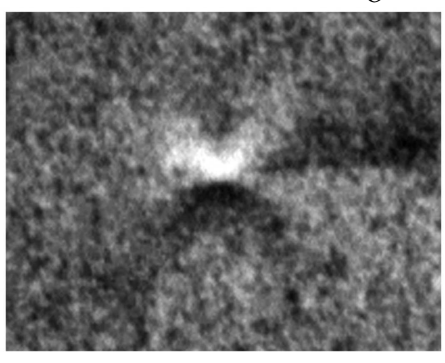

$c$

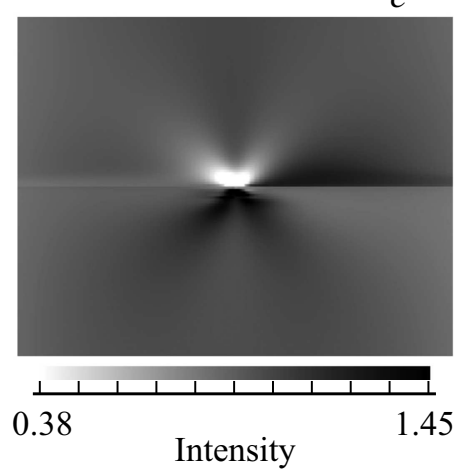

Рис. 4. $a)$ - контуры постоянных разориентаций $\beta_{h}$; $\left.b\right)$ - экспериментальное проекционное изображение дислокации; $\left.c\right)-$ моделирование проекционного изображения дислокации.

эту область эффективной. Границы этой области явно не выражены и почти в пять раз уже ширины треугольника рассеяния.

На рис. 2 показано теоретическое распределение (аналитическое решение) интенсивности внутри треугольника рассеяния в случае аномального прохождения рентгеновского излучения. С теоретической точки зрения на кристалл падает сферическая волна, которая засвечивает всю дисперсионную поверхность, при увеличении толщины кристалла сужается угловой спектр рассеяния. В этом случае дисперсионная поверхность засвечена лишь частично. И мы наблюдаем промежуточный режим рассеяния между сферической и плоской волной.

На рис. 3 показаны фрагменты экспериментальных секционных топограмм при дискретных положениях дислокации. На всех изображениях дислокация нахо- дится в эффективной области рассеяния. На рис. 2 видно, что за пределами этой области интенсивность излучения практически равна нулю. Область кристалла вблизи линии дислокации за пределами эффективной области не освещена падающим пучком, так как падающий пучок уже поглотился. Изменение положения дислокации осуществлялось путем смещения кристалла и фотопластинки относительно точки входа излучения в образец. Моделирование секционных изображений по уравнениям Такаги передает все характерные черты экспериментального изображения прямолинейной дислокации [14]. На рис. 3 и 4 для каждого фрагмента ширина $360 \mu \mathrm{m}$, высота $280 \mu \mathrm{m}$.

Рентгеновский пучок, падающий на исследуемый кристалл, имеет определенный угловой и хроматический спектр, связанный с характеристиками источника излу- 
чения и шириной щели $S_{2}$. Совершенный кристалл в условиях динамической дифракции взаимодействует с падающим пучком в узкой угловой области. Пусть в кристалле имеются единичные дефекты, тогда в случае тонкого кристалла изображение дефектов определяется двумя типами контраста: динамическим и кинематическим. Динамический контраст может наблюдаться от слабо искаженной области вдали от ядра дефекта. Кинематическое рассеяние возникает от искаженных областей кристалла и определяется более широким угловым и хроматическим спектром падающей волны и, как следствие, выделяется по интенсивности. Кинематическое изображение переносится на выходную поверхность образца с нормальным коэффициентом поглощения $P$ вблизи направления дифрагированной волны. Кинематическое изображение, рассеянное фрагментом искаженной решетки, привязано к локальной области образца и, соответственно, воспроизводится на топограммах сканирования.

В случае аномального прохождения рентгеновского излучения первая волна сильно поглощается и не принимает участия в дифракции. Кинематические изображения дефектов находящихся в объеме кристалла также исчезают и наблюдаются только дефекты расположенные вблизи выходной поверхности образца.

Рассмотрим рис. 3,a. Дислокация находится в левой части треугольника рассеяния и освещена интенсивным проходящим пучком. Слева от центра расположена область пониженной интенсивности, справа выброс интенсивности в виде цепочки интерференционных полос. На рисунках от $3, f$ до $3, e$ видно, как меняется изображение дислокации при смещении ее от края эффективной области к центру. На рис. 3, е дислокация расположена в центре треугольника рассеяния и изображение в целом симметрично относительно оси $Y$. На рис. $3, f$ дислокация находится в правой части треугольника рассеяния и освещена интенсивным отраженным пучком. Поэтому выброс интенсивности виден в направлении проходящего пучка. Изображения дислокации, расположенной на равных расстояниях от центра, $3, a$ и $3, f$ на экспериментальных топограммах почти зеркально симметричны относительно оси $Y$.

На рис. 3, е дислокация находится в центре треугольника рассеяния. Видно, что изображение имеет три темных лепестка и три светлых. На рис. 4, $a$ представлена карта локальных отклонений кристаллической решетки ( $\beta_{h}=$ const, формула (3)) от точного условия Брэгга. Она представляет собой четырехлепестковую розетку, два лепестка светлых, два темных. Видно, что распределение интенсивности на секционной топограмме на качественном уровне отличается от карты разориентаций. Они различаются как по распределению интенсивности, так и по симметрии рисунков. В целом секционное изображение и четырехлепестковая розетка это совершенно разные изображения.

Экспериментальное проекционное изображение является результатом сложения секционных изображений дислокации при разных ее положениях в эффективной области треугольника рассеяния. Проекционная топограмма имеет кинематический контраст от области сильных деформаций кристаллической решетки и динамический контраст от области слабых искажений. То и другое формируется упругими полями напряжений от дефекта и привязано к положению дефекта в объеме кристалла. На рис. 4, $b$ представлено проекционное изображение дислокации. Эта топограмма имеет более слабый контраст, чем секционные изображения на рис. 3. На ней размывается вся тонкая интерференционная структура секционных изображений.

В поляризационно-оптической и электронной микроскопии для краевой дислокации регистрируется розетка разориентаций. Ранее предполагалось, что такая же розетка будет наблюдаться и в методе проекционной топографии в случае аномального прохождения рентгеновского излучения [15-19]. Видно, что проекционное изображение на рис. $4, b$ согласуется в целом с розеткой разориентаций на рис. 4, $a$. Однако есть отличие, справа от ядра дислокации есть область повышенной интенсивности. Это связано с тем, что проекционное изображение формируется в объеме кристалла и освещается излучением при наклонном угле падения, в то время как розетка соответствует плоскому изображению деформаций.

\section{3. Моделирование дифракционного эксперимента и обсуждение}

Для теоретического описания распространения рентгеновского излучения в кристалле с произвольным типом дефектов используется система уравнений Такаги [20-23]. В двухволновом приближении, когда в кристалле распространяются две сильные волны проходящая и отраженная, система уравнений имеет вид

$$
\left\{\begin{array}{l}
\frac{\partial D_{o}}{\partial S_{o}}=-\pi K C \chi_{-h} D_{h} \\
\frac{\partial D_{h}}{\partial S_{h}}=-\pi K C \chi_{h} D_{o}+i 2 \pi K \beta_{h} D_{h} .
\end{array}\right.
$$

Здесь $D_{0}\left(s_{o}, s_{h}\right)$ и $D_{h}\left(s_{o}, s_{h}\right)-$ амплитуды прошедшей и дифрагированной волн, $K=1 / \lambda, \lambda$ - длина волны, $\chi_{h}$, $\chi_{-h}$ - комплексные коэффициенты Фурье-разложения поляризуемости кристалла, $\chi_{h}=\chi_{h r}+i \chi_{h i}, \mathbf{s}_{o}$ и $\mathbf{s}_{h}-$ единичные векторы вдоль направлений прошедшей и отраженной волн в кристалле, $C$ - поляризационный множитель, равный 1 и $|\cos 2 \vartheta|$ для $\sigma$ и $\pi$-поляризации. Функция $\beta_{h}$ описывает локальные отклонения отражающих плоскостей вблизи дефекта и связана с полем смещений внутри кристалла $\mathbf{u}(x, y)$ соотношением

$$
\beta_{h}=-\Delta \vartheta \sin 2 \vartheta-\frac{1}{K} \frac{\partial(\mathbf{h u})}{\partial s_{h}}
$$

где $\Delta \vartheta-$ угловое отклонение падающей волны от точного угла Брэгга. 
Функция $\beta_{h}$ описывает полную локальную деформацию кристаллической решетки вблизи дефекта локальные изменения параметра решетки и ее локальную разориентацию. Условие применимости системы уравнений (2) в случае динамического рассеяния: амплитуда волн в кристалле должна быть медленно меняющейся функцией на характерном расстоянии $L=\sqrt{\lambda \Lambda}$, где $\Lambda=\lambda \cos \theta_{B} / C \chi_{h r}$ - экстинкционная длина $[21,24]$. В рассматриваемом случае $\Lambda=15.46 \mu \mathrm{m}$. В рентгеновском диапазоне длин волн характерное расстояние порядка $0.05 \mu \mathrm{m}$. Это значение намного меньше разрешающей способности в методах рентгеновской дифракционной топографии.

В работе при решении задачи (1) численным методом использовалась дислокационная модель ПайерлсаНабарро [25,26]. Для этой модели выражение для $U_{x}$ описывающее поле смещений от прямолинейной краевой дислокации в периодической среде имеет вид

$$
U_{x}=\frac{b}{2 \pi}\left\{\operatorname{arctg} \frac{y}{x}+\frac{x y}{2(1-v)\left(x^{2}+y^{2}\right)}\right\}+\frac{b}{4} \operatorname{sign}(x),
$$

здесь $b$ - вектор Бюргерса, $v$ - коэффициент Пуассона.

При расчетах изображений дислокации приведение системы (2) к уравнениям в конечных разностях осуществлялось неявным методом второго порядка точности $[27,28]$.

Для анализа экспериментальных топограмм были рассчитаны секционные топограммы при различных расположениях дислокации в треугольнике рассеяния. Проекционная топограмма рассчитывались двумя методами: прямым интегрированием т.е. суммированием секционных изображений и второе с применением теоремы взаимности [29-31]. Применение теоремы взаимности позволяет сушественно уменьшить шаг интегрирования [31]. В дальнем поле дислокации шаг интегрирования вдоль оси $Z$ был равен $\Delta z=1 \mu \mathrm{m}$, а при $|y|<30 \mu \mathrm{m}$ $\Delta z=0.1 \mu \mathrm{m}$. На рис. 4, $c$ представлен результат численного интегрирования, с шагом вдоль оси $Y \Delta y=2 \mu \mathrm{m}$. Степень почернения на изображении пропорциональна логарифму интенсивности дифрагированного излучения. На топограмме численного моделирования в центральной части изображения можно увидеть четырехлепестковую розетку разориентаций и справа от центра область повышенной интенсивности. Что соответствует экспериментальной проекционной топограмме на рис. 4, $b$. На численной топограмме есть достаточное число мелких деталей, которые не видны на экспериментальной топограмме, так как есть дополнительное размытие связанное с конечным разрешением оптической схемы эксперимента и фотоматериала $17 \mu \mathrm{m}$.

В классическом представлении прямое или кинематическое изображение дислокации присутствует на секционных топограммах только в случае, когда сильно искаженная область вблизи ядра дислокации с быстро меняющимся полем разориентаций пересекает падающий пучок. Это связано с тем, что при динамической дифракции более широкий угловой и хроматический спектр содержатся только в падающем пучке. Динамические и кинематические волны рассеиваются под разными углами и не взаимодействуют друг с другом. В наших исследованиях все представленные топограммы дислокации были получены в случае, когда дислокация находилась в эффективной области рассеяния при аномальном распространении.

Для объяснения этого явления мы вводим понятие вторичного источника. Рассеяние излучения происходит на атомах. Атом поглощает фотон и через короткое время с определенной вероятностью испускает другой фотон со сферическим угловым спектром, с той же длиной волны и шириной спектра $\Delta \lambda / \lambda$, равной ширине соответствующей характеристической линии. Что касается коэффициента выхода вторичного излучения оно мало для легких материалов и имеет достаточно большое значение уже для средних атомов. Так, например, для $\mathrm{Si}$ вероятность выхода излучения составляет $4.3 \%$, а для германия уже $55.4 \%$.

При рассеянии излучения на дефекте совокупность атомов формирует квазимонохроматическую сферическую волну, что в сумме составляет вторичный некогерентный протяженный источник. Поскольку рассматриваются дефекты расположенные близко к выходной поверхности кристалла, то их кинематическое изображение регистрируется на топограмме. Просматривается аналогия с рассеянием в тонком кристалле. Изображения получаются протяженными, поскольку краевая дислокация является линейным объектом.

\section{4. Заключение}

В работе проанализированы особенности формирования изображения краевой дислокации, расположенной перпендикулярно поверхности образца в случае аномального прохождения рентгеновского излучения.

Анализ экспериментальных и расчетных изображений, приведенных в работе, позволяет сделать следующие выводы:

В работе предложен новый механизм формирования изображения дефектов в случае аномального прохождения рентгеновского излучения.

Проекционное изображение краевой дислокации в поглощающем кристалле в целом не имеет элементов симметрии и состоит из центральной части, которая соответствует розетке эффективных разориентаций и кинематического изображения дислокационной линии.

Изображение краевой дислокации на секционной топограмме зависит от ее положения в треугольнике рассеяния и ориентации вектора Бюргерса. В поглощающем кристалле изображение дислокации не имеет элементов симметрии. Контраст изображения краевой дислокации в проекционной топографии существенно ниже, чем в секционной топографии. 


\section{Финансирование работы}

Работа выполнена в рамках государственного задания ИФТТ РАН, ИПТМ РАН.

\section{Конфликт интересов}

Авторы заявляют, что у них нет конфликта интересов.

\section{Список литературы}

[1] N. Kato, A.R. Lang. Acta Cryst. A 12, 178 (1959).

[2] A. Authier. Dynamical Theory of $X$-Ray Diffraction. Oxford Science Publications (2001). $661 \mathrm{p}$.

[3] Э.В. Суворов, И.А. Смирнова. Успехи физических наук 185, 9, 897 (2015).

[4] Е.В. Шулаков, И.А. Смирнова, Э.В. Суворов. Поверхность. Рентген., синхротр. и нейтр. исслед. 7, 32 (1996).

[5] Е.В. Шулаков, И.А. Смирнова, Э.В. Суворов. Поверхность. Рентген., синхротр. и нейтр. исслед. 1, 101, (2002).

[6] В.Л. Инденбом, Ф.Н. Чуховский. Кристаллография 19, 1 , 35 (1974).

[7] В.Л. Инденбом, И.Ш. Слободецкий. Кристаллография 19 , 1, 42 (1974).

[8] И.А. Смирнова, Е.В. Шулаков, Э.В. Суворов, О.П. АлежкоОжевский. Поверхность. Рентген., синхротр. и нейтр. исслед. 1, 99 (2000).

[9] Э.В. Суворов, И.Л. Шульпина. Поверхность. Рентген., синхротр. и нейтр. исслед. 7, 3 (2001).

[10] Э.В. Суворов, И.А. Смирнова, Е.В. Шулаков. Поверхность. Рентген., синхротр. и нейтр. исслед. 9, 64 (2004).

[11] Э.В. Суворов, И.А. Смирнова. ФТТ 52, 241 (2010).

[12] Э.В. Суворов, И.А. Смирнова. ФТТ 52, 2325 (2010).

[13] V.G. Kohn, I.A. Smirnova. Acta Crystallogr. A 71, 519 (2015).

[14] И.А. Смирнова, Э.В. Суворов, Е.В. Шулаков. ФТТ 49, 1050 (2007).

[15] Л.Н. Данильчук, Т.А. Смородина. ФТТ 7, 4, 1245 (1965).

[16] Л.Н. Данильчук, А.И. Георгиев. Кристаллография 11, 2, 349 (1966).

[17] Л.Н. Данильчук, В.И. Никитенко. ФТТ 9, 7, 2027 (1967).

[18] A.O. Okunev, G.A. Verozubova, E.M. Trukhanov, I.V. Dzjuba, P.R.J. Galtier, S.A. Said Hassani. J. Appl. Cryst. 42, 994 (2009).

[19] A.O. Okunev, G.A. Verozubova. J.Appl. Cryst. 48, 1228 (2015).

[20] S. Takagi. Acta Crystallogr. 15, 1311 (1962).

[21] S. Takagi. J. Phys. Soc. Jpn 26, 5, 1239 (1969).

[22] D. Taupin. Bull. Soc. Fran. Mineral. Cristallogr. 87, 469 (1964).

[23] A.M. Afanas'ev, V.G. Kohn. Acta Cryst A 27, 421 (1971).

[24] J. Hartwig. J. Phys. D 34, 70 (2001).

[25] R. Wit. Phys. Status Solidi 20, 567 (1967).

[26] Дж. Хирт, И. Лоте. Теория дислокаций. Атомиздат, М. (1972). $598 \mathrm{c}$.

[27] A. Authier, C. Malgrange. M. Tournarie. Acta Cryst. A 24, 126 (1968).

[28] Y. Epelboin. J. AppI. Cryst. 7, 372 (1974).

[29] N. Като. Acta Cryst. А 24, 157 (1968).

[30] P.V. Petrashen, F.N. Chukhovskii, I.L. Shulpina. Acta Cryst. A 36, 287 (1980).

[31] C.A.M. Carvalho, Y. Epelboin. Acta Cryst. A 49, 467 (1993).

Редактор Т.Н. Василевская 